\title{
Imbedding Lie Algebras in Strongly Prime Algebras
}

\author{
José A. Anquela ${ }^{1}$, Teresa Cortés \\ anque@orion.ciencias.uniovi.es, cortes@orion.ciencias.uniovi.es \\ Departamento de Matemáticas, Universidad de Oviedo, \\ C/ Calvo Sotelo s/n, 33007 Oviedo, Spain \\ Telephone number: ++34985103185 \\ Fax number: ++34985102886
}

\begin{abstract}
We show that any Lie algebra with a sufficiently good module structure imbeds in a strongly prime Lie algebra with simple nondegenerate heart, spanned as a direct sum by the isomorphic image of the original algebra and the heart.
\end{abstract}

Keywords: Lie algebra, associative algebra, simple, strongly prime, heart

2010 Math. Subj. Class.: 16W10, 17B05, 17B60.

\section{Introduction}

In [2], we showed how to "paste" a simple heart to an arbitrary associative system over a field, so that it imbeds is a strongly prime (indeed primitive) system with nonzero simple primitive heart. This was aimed at building counterexamples linked to some problems with Jordan systems [1]. Recently, in [3], the results obtained in [2] were improved in two senses: on the one hand, associative systems over more general rings of scalars were considered, and, on the other hand, analogues for systems with involution were also obtained.

In this paper we will prove similar results for Lie algebras, so showing that the class of strongly prime Lie algebras with simple nondegenerate heart is wide enough to contain full information on the whole class of Lie algebras, at least when we work with sufficiently regular modules.

We will base our results on those for associative algebras, using Herstein's theorems $[6,7]$ on the transfer of regularity between associative algebras and the Lie algebras built out of them, together with Poincare-Birkhoff-Witt Theorem [9, Cor. $17.3 \mathrm{~B} ; 10$, Cor. 1 on page 160].

1 Corresponding author. 
The paper is divided into three sections, plus an Introduction in which basic results and terminology will be recalled. The first section is devoted to study centralizers and absolute zero divisors of subsets of endomorphisms acting densely on vector spaces. This sets appear naturally in the constructions of [2] and [3] for associative algebras. The results of Section 1 will be applied to give in the next section a modified version of the main construction of [2] for associative algebras over fields, specifically prepared to deal comfortably with nondegeneracy when applying it to Lie algebras. From that we will obtain a Lie analogue of the main theorem of [3] for Lie algebras over fields. In the final third section, we will obtain our main theorems, which match the associative analogues of [3]: we will find a necessary condition on the underlying module of a Lie algebra $L$ to find a prime Lie algebra where $L$ imbeds and see that the condition is also sufficient. Indeed, it guaranties that $L$ imbeds in a strongly prime Lie algebra with simple nondegenerate heart, spanned as a direct sum by the isomorphic image of $L$ and the heart.

\section{Preliminaries}

0.1 We will deal with associative and Lie algebras over an arbitrary ring of scalars $\Phi$. The reader is referred to $[8,9,10]$ for basic facts and notions not explicitly mentioned in this section.

0.2 A Lie algebra $L$ is said to be prime, if there are no nonzero orthogonal ideals of $L$ : if $I, K$ are nonzero ideals of $L$, then $[I, K] \neq 0$. An element $a \in L$ is said to be an absolute zero divisor of $L$ if $\left(\operatorname{ad}_{a}\right)^{2}=0$, i.e., $[a,[a, L]]=0$. A Lie algebra $L$ will be said nondegenerate if it does not have nonzero absolute zero divisors, and will be said strongly prime if it is prime and nondegenerate.

0.3 Given an associative or Lie algebra $M$, the heart Heart $(M)$ of $M$ is the intersection of all nonzero ideals of $M$. It can be readily seen that, when $R$ is a semiprime associative algebra, $\operatorname{Heart}(R)$ is the unique simple ideal of $R$ when it is nonzero.

0.4 An associative algebra $R$ gives rise to a Lie algebra $R^{(-)}$by antisymmetrization: over the same $\Phi$-module, the Lie product is given by $[x, y]=x y-y x$.

0.5 Let $R$ be an associative algebra over $\Phi$ without 2 -torsion. If $R$ is semiprime, then $R^{(-)} / Z(R)$ is nondegenerate [5, 4.2].

0.6 (i) Let $M$ be a $\Phi$-module. The annihilator of $M$ in $\Phi, \operatorname{Ann}_{\Phi}(M):=\{\lambda \in$ $\Phi \mid \lambda M=0\}$, is the kernel of the natural ring homomorphism of $\Phi$ in $\operatorname{End}_{\mathbf{z}}(M)$. Let

$\bar{\Phi}$ denote the quotient $\Phi / \operatorname{Ann}_{\Phi}(M)$. Notice that $\bar{\Phi}$ is isomorphic to the image of $\Phi$ 
in $\operatorname{End}_{\mathbf{Z}}(M)$ and $M$ becomes a $\bar{\Phi}$-module. Moreover, if $M$ is an associative or Lie algebra over $\Phi$, then it is also an associative or Lie algebra, respectively, over $\bar{\Phi}$.

(ii) Conversely, if we take any proper ideal $I$ of $\Phi$, any $\Phi / I$-module, or associative or Lie algebra over $\Phi / I$ can be viewed as a $\Phi$-module, or associative or Lie algebra over $\Phi$, respectively, in the obvious manner.

0.7 For a vector space $V$ over a division $\operatorname{ring} F, \operatorname{End}_{F}(V)_{0}$ will denote the ideal of $\operatorname{End}_{F}(V)$ consisting of the endomorphisms of $V$ with finite rank. It is well known that $\operatorname{End}_{F}(V)_{0}$ is a simple associative ring, which is left primitive since it acts densely on $V$ on the left.

This paper is aimed at studying Lie analogues of the following two results for associative algebras.

0.8 If $R$ is an associative system over $\Phi$, and there exists a prime associative system $\tilde{R}$ over $\Phi$ such that $R$ is isomorphic to a subsystem $S$ of $\tilde{R}$, then $\bar{\Phi}$ is an integral domain acting without torsion on $R$ [3, 2.1].

0.9 Let $R$ be an associative algebra over a ring of scalars $\Phi$ such that $\bar{\Phi}$ is an integral domain acting without torsion on $R$. There exists an associative algebra $\tilde{R}$ over $\Phi$ such that:

(i) $R$ is isomorphic to a subalgebra $S$ of $\tilde{R}$,

(ii) $\tilde{R}$ is a left primitive algebra, hence it is prime,

(iii) Heart $(\tilde{R})$ is simple and left primitive,

(iv) $\tilde{R}=S \oplus \operatorname{Heart}(\tilde{R})$, hence $\tilde{R} / \operatorname{Heart}(\tilde{R}) \cong R[3,2.2]$.

\section{Lie Algebras Built out of Dense Sets of Endomorphisms}

In this section we will deal with vector spaces over a division ring. Though for later use in the paper working with vector spaces over fields would be enough, the generalization does not involve much extra work and the results we obtain might be applied to Lie algebras built out of primitive rings, without restrictions.

1.1 If $V$ is a left vector space over a division $\operatorname{ring} F$, a subset $S \subseteq \operatorname{End}_{F}(V)$ is said to be dense on $V$ (or to act densely on $V$ ) if, for any $n \in \mathbf{N}$ and any $v_{1}, \ldots, v_{n}, w_{1}, \ldots, w_{n} \in V$ such that $v_{1}, \ldots, v_{n}$ are linearly independent, there exists $f \in S$ such that $f\left(v_{i}\right)=w_{i}$ for all $i=1, \ldots, n$.

The next result will give us full information on the endomorphisms of a vector space commuting with a subset acting densely on the vector space. It is the starting 
point to find information on elements acting as absolute zero divisors on these dense sets.

1.2 Proposition. If $V$ is a left vector space over a division ring $F$, and $S$ is a subset of $\operatorname{End}_{F}(V)$ acting densely on $V$, then an element of $\operatorname{End}_{F}(V)$ commuting with all the elements of $S$ is necessarily a scalar multiple of the identity by a central element of $F$, i.e.,

$$
C_{\operatorname{End}_{F}(V)}(S)=Z(F) \cdot \operatorname{Id}_{V}
$$

As a consequence, if, in addition, $S$ is a subring of $\operatorname{End}_{F}(V)$ or a Lie subring of $\operatorname{End}_{F}(V)^{(-)}$, then $Z(S)=S \cap Z(F) \cdot \operatorname{Id}_{V}$.

Proof: For any $f \in C_{\operatorname{End}_{F}(V)}(S)$, and any $0 \neq v \in V, v$ and $f(v)$ are linearly dependent over $F$. Otherwise, by density, we can find $g \in S$ such that $g(v)=0$ and $g(f(v))=v$. Thus, $v=g(f(v))=f(g(v))=f(0)=0$ since $f$ and $g$ commute, which is a contradiction. We have shown that, given $f \in C_{\operatorname{End}_{F}(V)}(S)$, for any $0 \neq v \in V$ there exists $\lambda_{v} \in F$ (obviously unique for each $0 \neq v \in V$ ) such that $f(v)=\lambda_{v} v$.

Moreover, for any $0 \neq v \in V, \lambda_{v} \in Z(F)$. Indeed, by density, for any $0 \neq v \in V$, and any $\alpha \in F$, there exists $h \in S$ such that $h(v)=\alpha v$, hence $\alpha \lambda_{v} v=\alpha f(v)=f(\alpha v)$ (by $F$-linearity of $f)=f(h(v))=h(f(v))=h\left(\lambda_{v} v\right)=\lambda_{v} h(v)$ (by $F$-linearity of $h$ ) $=\lambda_{v} \alpha v$, which implies that $\alpha \lambda_{v}=\lambda_{v} \alpha$, for any $\alpha \in F$, as desired.

The fact that $\lambda_{v} \in Z(F)$ implies that, for any $0 \neq v \in V$, and for any $0 \neq \alpha \in F$, $\lambda_{\alpha v}=\lambda_{v}: \lambda_{\alpha v} \alpha v=f(\alpha v)=\alpha f(v)$ (by $F$-linearity of $f$ ) $=\alpha \lambda_{v} v=\lambda_{v} \alpha v$ (since $\left.\lambda_{v} \in Z(F)\right)$ implies $\lambda_{\alpha v} \alpha=\lambda_{v} \alpha$, hence $\lambda_{\alpha v}=\lambda_{v}$. Also the equality

$$
\lambda_{v} v+\lambda_{w} w=f(v)+f(w)=f(v+w)=\lambda_{v+w}(v+w)
$$

applied to any pair or linearly independent elements $v, w \in V$ shows that the scalars $\lambda_{v}$ are all the same, i.e., $f=\lambda I d_{V}$, for some $\lambda \in Z(F)$.

1.3 Proposition. If $V$ is a left vector space over a division ring $F$ without 2-torsion, and $S$ is a subset of $\operatorname{End}_{F}(V)$ acting densely on $V$, then an element $f$ of $\operatorname{End}_{F}(V)$ such that $[f,[f, g]]=0$, for all $g \in S$ is necessarily a scalar multiple of the identity by a central element of $F$, i.e.,

$$
\left\{f \in \operatorname{End}_{F}(V) \mid[f,[f, S]]=0\right\}=Z(F) \cdot \operatorname{Id}_{V} .
$$

Proof: Let $f \in \operatorname{End}_{F}(V)$ satisfy $[f,[f, S]]=0$.

We claim that, for any $0 \neq v \in V, v$ and $f(v)$ are linearly dependent over $F$, so that there exists a unique $\lambda_{v} \in F$ such that $f(v)=\lambda_{v} v$. Otherwise, we have two possibilities: 
1) $v, f(v), f(f(v))$ are linearly independent, which implies, by density of $S$, that there is $g \in S$ such that $g(v)=0, g(f(v))=v$, and $g(f(f(v)))=0$. Now the fact that $0=[f,[f, g]]=f f g+g f f-2 f g f$ acting on $v$ yields $0=f f g(v)+g f f(v)-2 f g f(v)=$ $-2 f(v)$, hence $f(v)=0$ due to the absence of 2-torsion, which is a contradiction.

2) $v, f(v)$ are linearly independent but $f(f(v))$ lies in $W:=F\langle v, f(v)\rangle$, the vector subspace of $V$ spanned by $v$ and $f(v)$. Then $W$ is $f$-invariant, and we can consider $\tilde{f} \in$ $\operatorname{End}_{F}(W)$ obtained by restricting $f$ to $W$. By density of $S$, any element of $\operatorname{End}_{F}(W)$ can be obtained by restricting to $W$ a suitable element of $S$, hence $\left[\tilde{f},\left[\tilde{f}, \operatorname{End}_{F}(W)\right]\right]=$ 0 , i.e., $\tilde{f}$ is an absolute zero divisor of $\operatorname{End}_{F}(W)^{(-)}$. By (0.5) applied to the $\mathbf{Z}$ algebra $\operatorname{End}_{F}(W)$ (it is simple, hence prime, hence semiprime), $\tilde{f} \in Z\left(\operatorname{End}_{F}(W)\right)=$ $Z(F) \cdot I d_{W}$ (the last equality follows from (1.2) applied to the whole $\operatorname{End}_{F}(W)$, which acts densely on $W)$. Thus $f(v)=\tilde{f}(v)$ is a scalar multiple of $v$, which contradicts our assumption.

Moreover, for any $0 \neq v \in V, \lambda_{v} \in Z(F)$. Indeed, by density, for any $0 \neq$ $v \in V$, and any $\alpha \in F$, there exists $h \in S$ such that $h(v)=\alpha v$. Thus, using $F$ linearity of $f$ and $h$, the equality $[f,[f, h]](v)=(f f h+h f f-2 f h f)(v)=0$ reads $\left(\alpha \lambda_{v}^{2}+\lambda_{v}^{2} \alpha-2 \lambda_{v} \alpha \lambda_{v}\right) v=0$, hence $0=\alpha \lambda_{v}^{2}+\lambda_{v}^{2} \alpha-2 \lambda_{v} \alpha \lambda_{v}=\left[\lambda_{v},\left[\lambda_{v}, \alpha\right]\right]$, and $\lambda_{v}$ is an absolute zero divisor of $F^{(-)}$. By (0.5) applied to the ring $F$ (it is a division ring, hence it is semiprime), $\lambda_{v} \in Z(F)$.

Now the arguments of the last paragraph of the proof of (1.2) apply here verbatim to show that $f=\lambda I d_{V}$, for some $\lambda \in Z(F)$.

1.4 Lemma. If $V$ is a left vector space of infinite dimension over a division ring $F$, and $S$ is a subset of $\operatorname{End}_{F}(V)$ acting densely on $V$, then the set $[S, S]:=$ $\{[f, g] \mid f, g \in S\}$ acts densely of $V$ too.

Proof: Let $v_{1}, \ldots, v_{n}, w_{1}, \ldots, w_{n} \in V, n \in \mathbf{N}$, such that $v_{1}, \ldots, v_{n}$ are linearly independent. We will show that there exist $f, g \in S$ such that

$$
[f, g]\left(v_{i}\right)=f\left(g\left(v_{i}\right)\right)-g\left(f\left(v_{i}\right)\right)=w_{i}, \quad \text { for any } i \in\{1, \ldots, n\}
$$

Since $V$ is infinite-dimensional, we can find $u_{1}, \ldots, u_{n} \in V$ such that $v_{1}, \ldots, v_{n}$, $u_{1}, \ldots, u_{n}$ are linearly independent. By density of $S$ on $V$, there exist $f, g \in S$ such that $f\left(v_{i}\right)=0$, for any $i \in\{1, \ldots, n\}$, and $f\left(u_{i}\right)=w_{i}$, for any $i \in\{1, \ldots, n\}$, and $g\left(v_{i}\right)=u_{i}$, for any $i \in\{1, \ldots, n\}$. Clearly, $f$ and $g$ satisfy (1).

1.5 Corollary. If $V$ is a left vector space of infinite dimension over a division ring $F$ without 2-torsion, and $R=\operatorname{End}_{F}(V)_{0}$, then $\left\{f \in \operatorname{End}_{F}(V) \mid[f,[f,[R, R]]]=\right.$ $0\}=Z(F) \cdot \operatorname{Id}_{V}$. As a consequence, $C_{\operatorname{End}_{F}(V)}([R, R])=Z(F) \cdot \operatorname{Id}_{V}$, and $Z(R)=0$.

Proof: By (0.7) and (1.4), $[R, R]$ acts densely on $V$. We just need to use (1.3) applied to $[R, R]$, together with the clear fact that $R \cap Z(F) \cdot \operatorname{Id}_{V}=0$. 


\section{Growing Hearts in Lie Algebras over Fields}

2.1 Let $L$ be a Lie algebra and $L_{1}$ be a subalgebra of $L$ containing an ideal $I$ of $L$. If $C_{L}(I)=0$, then $I$ hits any nonzero ideal of $L_{1}$ : if $K$ is an ideal of $L_{1}$ and $K \cap I=0$, then $[K, I] \subseteq K \cap I=0$, and $K \subseteq C_{L}(I)=0$. If, in addition, $I$ is simple, then $I$ is contained in every nonzero ideal of $L_{1}$ (if $K$ is a nonzero ideal of $L_{1}$, then $0 \neq K \cap I$ is an ideal of $I$, hence $I=K \cap I \subseteq K$ using the simplicity of $I$ ), i.e., $I=\operatorname{Heart}\left(L_{1}\right)$.

In order to be able to obtain our main theorem for Lie algebras, we need a modified version of $[2,2.3]$ for algebras, in which the simple Lie ideal associated to the associative heart has zero centralizer and there is no nonzero element acting on it as an absolute zero divisor.

2.2 TheOREM. Let $R$ be an associative algebra over a field $F$ of characteristic different from 2. There exists an associative algebra $\tilde{R}$ over $F$ such that:

(i) $R$ is isomorphic to a subalgebra $S$ of $\tilde{R}$,

(ii) $\tilde{R}$ is a left primitive algebra, hence it is prime,

(iii) $\operatorname{Heart}(\tilde{R})$ is simple and left primitive,

(iv) $\tilde{R}=S \oplus \operatorname{Heart}(\tilde{R})$, hence $\tilde{R} / \operatorname{Heart}(\tilde{R}) \cong R$.

(v) $C_{\tilde{R}}([\operatorname{Heart}(\tilde{R}), \operatorname{Heart}(\tilde{R})])=0$

(vi) If $a \in \tilde{R}$ satisfies $[a,[a,[\operatorname{Heart}(\tilde{R}), \operatorname{Heart}(\tilde{R})]]]=0$ then $a=0$.

ProOF: We just need to modify the construction given in the proof of $[2,2.3]$, so that the additional conditions (v) and (vi) hold:

Let $V=\Sigma_{i \in \mathbf{N}} V_{i}$, where $V_{i}=\hat{R}$, for all $i \in \mathbf{N}$, and $\tau_{i}: \hat{R} \longrightarrow V, i \in \mathbf{N}$, be the canonical injections. Let $\Psi: R \longrightarrow \operatorname{End}_{F}(V)$ be the map given by $\Psi(a) \cdot \tau_{i}=\tau_{i} \cdot L_{a}$ ( $L_{a}$ is the left multiplication by $a$ in $\hat{R}$ ) if $i$ is odd, and $\Psi(a) \cdot \tau_{i}=0$ if $i$ is even. It is easy to see that $\Psi$ is an $F$-algebra monomorphism, so that $S:=\Psi(R)$ is a subalgebra of $\operatorname{End}_{F}(V)$ isomorphic to $R$. Moreover,

$$
S \cap \operatorname{End}_{F}(V)_{0}=0
$$

since, given any $0 \neq x \in R, \tau_{i}(x)=\Psi(x)\left(\tau_{i}(1)\right)$ for any odd $i$, which implies $\Psi(x) \notin$ $\operatorname{End}_{F}(V)_{0}$.

Take $\tilde{R}=S+\operatorname{End}_{F}(V)_{0}$, which by (1) is a direct sum (of a subalgebra and an ideal of $\operatorname{End}_{F}(V)(0.7)$ ), hence it is a subalgebra of $\operatorname{End}_{F}(V)$. Moreover, $\operatorname{End}_{F}(V)_{0}$ is an ideal of $\tilde{R}$. Since $\operatorname{End}_{F}(V)_{0}$ acts densely on the left on $V$, so does $\tilde{R}$, hence $\tilde{R}$ is left primitive and, in particular, it is prime. Now $\operatorname{End}_{F}(V)_{0}$ is a simple ideal of 
the prime associative algebra $\tilde{R}$, so that $\operatorname{Heart}(\tilde{R})=\operatorname{End}_{F}(V)_{0}$, which is simple and left primitive. This shows (i-iv).

Notice that

$$
\tilde{R} \cap F \cdot \operatorname{Id}_{V}=0
$$

Indeed, given $x \in R$, and $f \in \operatorname{End}_{F}(V)_{0}$, we claim that $\Psi(x)+f$ is not surjective. Otherwise $V=\operatorname{Im}(\Psi(x)+f) \subseteq \operatorname{Im}(\Psi(x))+\operatorname{Im} f$ implies $V=\operatorname{Im}(\Psi(x))+\operatorname{Im} f$, hence $\operatorname{Im}(\Psi(x))$ has finite codimension in $V(V / \operatorname{Im}(\Psi(x)) \cong \operatorname{Im} f /(\operatorname{Im}(\Psi(x)) \cap \operatorname{Im} f)$ is finite dimensional), which is a contradiction since $\sum_{\text {even } i} \tau_{i}(\hat{R})$ is an infinite-dimensional subspace of $V$ intersecting trivially $\operatorname{Im}(\Psi(x))$. Since $\lambda \operatorname{Id}_{V}$ is even bijective for any $\lambda \in F \backslash\{0\},(2)$ is clear.

Now

$$
\begin{aligned}
C_{\tilde{R}}([\operatorname{Heart}(\tilde{R}), \operatorname{Heart}(\tilde{R})]) & =C_{\tilde{R}}\left(\left[\operatorname{End}_{F}(V)_{0}, \operatorname{End}_{F}(V)_{0}\right]\right) \\
& =C_{\operatorname{End}_{F}(V)}\left(\left[\operatorname{End}_{F}(V)_{0}, \operatorname{End}_{F}(V)_{0}\right]\right) \cap \tilde{R} \\
& =F \cdot \operatorname{Id}_{V} \cap \tilde{R}(\operatorname{by}(1.5))=0
\end{aligned}
$$

by (2), which shows (v).

Finally, (vi) follows from (1.5) and (2).

We will use (2.2) to obtain an analogue of (0.9) for Lie algebras over a field of characteristic not two.

2.3 Proposition. Let $L$ be an Lie algebra over a field $F$ of characteristic not two. There exists a Lie algebra $\tilde{L}$ over $F$ such that:

(i) $L$ is isomorphic to a subalgebra $M$ of $\tilde{L}$,

(ii) $\tilde{L}$ is strongly prime,

(iii) Heart $(\tilde{L})$ is simple and nondegenerate,

(iv) $\tilde{L}=M \oplus \operatorname{Heart}(\tilde{L})$, hence $\tilde{L} / \operatorname{Heart}(\tilde{L}) \cong L$.

(v) $C_{\tilde{L}}(\operatorname{Heart}(\tilde{L}))=0$ and there is not any $0 \neq a \in \tilde{L}$ such that $[a,[a$, Heart $(\tilde{L})]]=0$.

Proof: By the Poincare-Birkhoff-Witt Theorem [9, Cor. 17.3 B; 10, Cor. 1 on page 160], $L$ is a subalgebra of $R^{(-)}$for some associative algebra $R$ over $F$. Let $\tilde{R}$ be the algebra whose existence is given in (2.2), so that $S$ is a subalgebra of $\tilde{R}$ isomorphic to $R$. Let $M$ be the image of $L$ through the isomorphism $R \cong S$. We have that $M$ is a subalgebra of $\tilde{R}^{(-)}$not hitting $H:=\operatorname{Heart}(\tilde{R})$. Clearly, $H:=\operatorname{Heart}(\tilde{R})$ is an ideal of $\tilde{R}^{(-)}$, and so is $[H, H]$. Therefore $\tilde{L}:=M \oplus[H, H]$ is a subalgebra of $\tilde{R}^{(-)}$and (i) holds. 
Notice that $C_{\tilde{R}}([H, H])=0(2.2)(\mathrm{v})$ implies $C_{\tilde{L}}([H, H])=0$ and $Z(H)=0$, hence $[H, H]$ is a simple Lie algebra by [7, Theorem 4]. Therefore (2.1) can be applied to obtain Heart $(\tilde{L})=[H, H]$, which implies (iv). Since $Z(\tilde{R})=0$ (again as a consequence of $\left.C_{\tilde{R}}([H, H])=0(2.2)(\mathrm{v})\right), \tilde{R}^{(-)}$is nondegenerate by $(0.5)$, and its ideal $[H, H]$ is nondegenerate by $[4$, Lemma $1.1 ; 11$, Lemma 4$]$, which shows (iii).

Because of having a simple heart, $\tilde{L}$ is obviously prime. Moreover, $\tilde{L}$ is nondegenerate by $(2.2)(\mathrm{vi})$, which proves (ii). Finally, (v) is a direct application of $(2.2)(\mathrm{v})(\mathrm{vi})$ too.

2.4 REMARK. In case we are working over fields of characteristic zero, we can use Zelmanov's result [11, Cor. 1 on page 543], to show directly nondegeneracy of $\tilde{L}$ in (2.3) from the nondegeneracy of its heart: if $K($ ) denotes the Kostrikin radical (the smallest ideal giving a nondegenerate quotient) $0=K(\operatorname{Heart}(\tilde{L}))=K(\tilde{L}) \cap \operatorname{Heart}(\tilde{L})$ implies $K(\tilde{L})=0$, i.e., $\tilde{L}$ is nondegenerate.

\section{Main Results}

The aim of this section is obtaining an optimal version of (2.3), for Lie algebras over more general rings of scalars. The next result sets up a limit on this generalization.

3.1 Proposition. If $L$ is a Lie algebra over a ring of scalars $\Phi$, and there exists a prime Lie algebra $\tilde{L}$ over $\Phi$ such that $L$ is isomorphic to a subalgebra $M$ of $\tilde{L}$, then $\bar{\Phi}$ is an integral domain acting without torsion on $L$.

Proof: Let $\lambda+\operatorname{Ann}_{\Phi}(L), \mu+\operatorname{Ann}_{\Phi}(L) \in \bar{\Phi}$. If $\left(\lambda+\operatorname{Ann}_{\Phi}(L)\right)\left(\mu+\operatorname{Ann}_{\Phi}(L)\right)=0$, then the ideals of $\tilde{L}$ generated by $\lambda M$, and $\mu M$ are orthogonal. By primeness of $\tilde{L}$, either $\lambda M=0$ or $\mu M=0$, i.e., either $\lambda L=0$ or $\mu L=0$, i.e., $\lambda+\operatorname{Ann}_{\Phi}(L)=0$ or $\mu+\operatorname{Ann}_{\Phi}(L)=0$.

If $\lambda+\operatorname{Ann}_{\Phi}(L) \in \bar{\Phi}$ and $r \in L$ satisfy $\lambda r=0$, then $\lambda s=0$, for the image $s$ of $r$ under the isomorphism $L \cong M$. Now, the ideals of $\tilde{L}$ generated by $s$ and $\lambda \tilde{L}$ are orthogonal. By primeness of $\tilde{L}$, either $s=0$, i.e., $r=0$, or $\lambda \tilde{L}=0$, which implies $\lambda M=0$, i.e., $\lambda L=0$, i.e., $\lambda+\operatorname{Ann}_{\Phi}(L)=0$.

We are going to see that the converse of the above result is true. In fact, it is part of the announced optimal version of (2.3).

3.2 TheOREM. Let $L$ be a Lie algebra over a ring of scalars $\Phi$ such that $\bar{\Phi}$ is an integral domain acting without torsion on L. Let us also assume that $L$ (equivalently $\bar{\Phi})$ does not have 2-torsion. There exists a Lie algebra $\tilde{L}$ over $\Phi$ such that:

(i) $L$ is isomorphic to a subalgebra $M$ of $\tilde{L}$, 
(ii) $\tilde{L}$ is strongly prime,

(iii) Heart $(\tilde{L})$ is simple and nondegenerate,

(iv) $\tilde{L}=M \oplus \operatorname{Heart}(\tilde{L})$, hence $\tilde{L} / \operatorname{Heart}(\tilde{L}) \cong L$.

(v) $C_{\tilde{L}}(\operatorname{Heart}(\tilde{L}))=0$ and there is not any $0 \neq a \in \tilde{L}$ such that $[a,[a, \operatorname{Heart}(\tilde{L})]]=0$.

Proof: First notice that we can replace $\Phi$ by $\bar{\Phi}$ and assume that $\Phi$ is an integral domain acting without torsion on $L$ ( $\Phi$ and $L$, both without 2-torsion): We just need to work over $\bar{\Phi}$ and then read the result in terms of $\Phi$-modules and $\Phi$-algebras (0.6)(ii). Isomorphisms, submodules, subalgebras, ideals, simplicity, primeness, nondegeneracy, etc. over $\bar{\Phi}$ are the same over $\Phi$, and $L$ goes back to its initial $\Phi$-module structure.

Now let $L_{1}:=\Phi^{-1} \Phi \otimes_{\Phi} L$, which is a Lie algebra over the field of fractions $F:=\Phi^{-1} \Phi$ of $\Phi$ of characteristic not two. If we apply (2.3) to $L_{1}$ as an $F$-algebra, we obtain an $F$-algebra $\tilde{L}_{1}$ satisfying $(2.3)(\mathrm{i}-\mathrm{v})$. Let $H:=\operatorname{Heart}\left(\tilde{L}_{1}\right)$. It is a simple nondegenerate $F$-algebra which is, in particular a simple nondegenerate $\Phi$-algebra (simplicity and the absence of absolute zero divisors do not depend on the ring of scalars).

Let $M_{1}$ be the isomorphic image of $L_{1}$ in $\tilde{L}_{1}$. Since $L$ is a $\Phi$-subalgebra of $L_{1}$ due to the lack of torsion, its image $M$ by the isomorphism $L_{1} \cong M_{1}$ is also a $\Phi$-subalgebra of $M_{1}$ (hence of $\tilde{L}_{1}$ ) isomorphic to $L$.

Now we can define $\tilde{L}:=M \oplus H\left(M \cap H \subseteq M_{1} \cap H=0\right)$ which is a $\Phi$-subalgebra of $\tilde{L}_{1}$ and hence satisfies (i) for $L$.

Since $\{a \in \tilde{L} \mid[a,[a, H]]=0\} \subseteq\left\{a \in \tilde{L}_{1} \mid[a,[a, H]]=0\right\}$, and $C_{\tilde{L}}(H) \subseteq C_{\tilde{L}_{1}}(H)$, $(2.3)(\mathrm{v})$ implies:

(1) $C_{\tilde{L}}(H)=0$ and there is not any $0 \neq a \in \tilde{L}$ such that $[a,[a, H]]=0$.

Moreover, $C_{\tilde{L}_{1}}(H)=0((2.3)(\mathrm{v}))$ allow us to apply $(2.1)$ to obtain $\operatorname{Heart}(\tilde{L})=H$, so that we have (iii) and (iv), and (1) is (v).

Because of having a simple heart, $\tilde{L}$ is prime, and its nondegeneracy follows from ( v)

3.3 Remark. Following the proof of (2.3) and its use to prove (3.2), an additional fact can be stressed: By (1.4), both $\tilde{L}$ and Heart $(\tilde{L})$ in (3.2) are subsets of $\operatorname{End}_{F}(V)$ acting densely on $V$, for an infinite dimensional vector space $V$ over the field of fractions $F$ of $\bar{\Phi}$.

Acknowledgements: Partly supported by the Ministerio de Educación y Ciencia and Fondos FEDER, MTM2007-62390, and by the Ministerio de Ciencia e Inno- 
vación and Fondos FEDER, MTM2010-16153, and I3 2010/00075/001.

\section{REFERENCES}

[1] J. A. Anquela, T. Cortés, "Local and Subquotient Inheritance of Simplicity in Jordan Systems" Journal of Algebra 240 (2001), 680-704.

[2] J. A. Anquela, T. Cortés, "Jordan cubes and Associative Powers", J. Pure Appl. Algebra 181 (2003), 131-139.

[3] J. A. Anquela, T. Cortés, "Growing Hearts in Associative Systems" (to appear). [available at the Jordan Theory Preprint Archives http://homepage.uibk.ac.at/ c70202/jordan/archive/growinghearts/index.html]

[4] E. García, "Inheritance of Primeness by Ideals in Lie Algebras", Int. J. Math., Game Theor. Algebra 13 (6) (2003), 481-884.

[5] E. García, M. Gómez-Lozano, "A Characterization of the Kostrikin Radical of a Lie Algebra" (to appear) [available at the Jordan Theory Preprint Archives http://homepage.uibk.ac.at/ ${ }^{\sim}$ 70202/jordan/archive/kostrikin-lie/index.html].

[6] I. N. Herstein, "On the Lie and Jordan Rings of a Simple Associative Ring", Amer J. Math. 77 (1955), 279-285.

[7] I. N. Herstein, "Lie and Jordan Structures in Simple Associative Rings", Bull. Amer. Math. Soc. 67 (1961), 517-531.

[8] I. N. Herstein, Noncommutative Rings, The Carus Mathematical Monographs, No. 15, The Mathematical Association of America, New York, 1968.

[9] J. E. Humphreys, Introduction to Lie Algebras and Representation Theory, Springer-Verlag, New York, 1980.

[10] N. Jacobson, Lie Algebras, Dover Publications, Inc., New York, 1979.

[11] E. Zelmanov, "Lie Algebras with an Algebraic Adjoint Representation", Math. USSR Sbornik 49 (2) (1984), 537-552. 